\title{
Erratum: Simple and tight monogamy relations for a class of Bell inequalities [Phys. Rev. A 95, 012113 (2017)]
}

\author{
Remigiusz Augusiak \\ (Received 5 September 2017; published 6 October 2017)
}

DOI: 10.1103/PhysRevA.96.049901

The correlations defined in Eq. (13) and in the following text do not saturate the Bell inequality $(9)$ in all scenarios $(N, m, d)$. The correct ones that do saturate (9) are bilocal correlations given by

$$
p_{A_{1} \cdots A_{N}}^{\mathrm{bi}}(\mathbf{a} \mid \mathbf{x})=p_{A_{1} \cdots A_{N-1}}\left(a_{1}, \ldots, a_{N-1} \mid x_{1}, \ldots, x_{N-1}\right) p_{A_{N}}\left(a_{N} \mid x_{N}\right),
$$

where $p_{A_{N}}\left(a_{N} \mid x_{N}\right)$ is a single-party probability distribution defined as $p_{A_{N}}\left(0 \mid x_{N}\right)=1$ for any $x_{N}$ whereas to introduce $p_{A_{1} \cdots A_{N-1}}$ we need to consider the case of even and odd $N$ 's. For even $N$ we have

$$
p_{A_{1} \cdots A_{N-1}}\left(\mathbf{a} \mid \alpha_{1}+1, \alpha_{1}+\alpha_{2}-1, \ldots, \alpha_{N-2}+\alpha_{N-1}-1\right)= \begin{cases}\frac{1}{d^{N-2}}, & \sum_{i=1}^{N-1}(-1)^{i-1} a_{i}=\widetilde{f}_{e}(\boldsymbol{\alpha}), \\ 0, & \text { otherwise, }\end{cases}
$$

whereas for odd $N$,

$$
p_{A_{1} \cdots A_{N-1}}\left(\mathbf{a} \mid \alpha_{1}, \alpha_{1}+\alpha_{2}-1, \ldots, \alpha_{N-2}+\alpha_{N-1}-1\right)= \begin{cases}\frac{1}{d^{N-2}}, & \sum_{i=1}^{N-1}(-1)^{i-1} a_{i}=\tilde{f}_{o}(\boldsymbol{\alpha}), \\ 0, & \text { otherwise }\end{cases}
$$

where $\alpha_{i}=1, \ldots, M$ for $i=1, \ldots, N-2$ and $\alpha_{N-1}=1, \ldots, M-1$. The factors $\widetilde{f_{o}}(\boldsymbol{\alpha})$ and $\tilde{f}_{e}(\boldsymbol{\alpha})$ are defined as

$$
\widetilde{f}_{\boldsymbol{o}}(\boldsymbol{\alpha})=\sum_{i=1}^{N-2}(-1)^{i+1} H\left(\alpha_{i}+\alpha_{i+1}-M-1\right),
$$

and $\widetilde{f}_{e}(\boldsymbol{\alpha})=\widetilde{f}_{o}(\boldsymbol{\alpha})-H\left(\alpha_{1}+1-M\right)$, where $H(x)$ is the step Heaviside function, that is, $H(x)=1$ for $x>0$ and $H(x)=0$ for $x \leqslant 0$.

For the remaining choices of measurements that are not included in Eqs. (2) and (3), for both odd and even $N$ 's, we simply assume that $p_{A_{1} \cdots A_{N-1}}\left(a_{1}, \ldots, a_{N-1} \mid x_{1}, \ldots, x_{N-1}\right)=1 / d^{N-1}$. Also, we remember that, whenever in Eqs. (2) or (3), $\alpha_{1}+1$ or $\alpha_{i}+\alpha_{i+1}-1$ for some $i$ exceeds $M$, one needs to subtract $M$ from it.

It is not difficult to see that the probability distribution $p_{A_{1} \cdots A_{N-1}}$ for both odd and even $N$ 's is nonsignaling as by tracing one of the parties one obtains a fully random $(N-2)$-partite probability distribution $1 / d^{N-2}$. Moreover, to see that $p_{A_{1} \cdots A_{N}}^{\text {bi }}(\mathbf{a} \mid \mathbf{x})$ saturates inequality (9) of the original article one uses the identities (39) and (47) proved therein.

Then, the correlations $p_{A_{1} \cdots A_{N}}^{\text {loc }}\left(a_{1}, \ldots, a_{N} \mid x_{1}, \ldots, x_{N}\right)$ appearing in Eq. (51) need to be replaced by $p_{A_{1} \cdots A_{N}}^{\text {bi }}\left(a_{1}, \ldots, a_{N} \mid x_{1}, \ldots, x_{N}\right)$ defined in Eq. (1), whereas $p_{A_{N+1}}\left(a_{N+1} \mid x_{N+1}\right)$ introduced in the text after Eq. (51) should be defined as $p_{A_{N+1}}\left(0 \mid x_{N+1}\right)=1$ for all choices of $x_{N+1}$.

Finally, the conditions in Eqs. (10) and (12) of the original paper should read

$$
\sum_{i=0}^{N-1}(-1)^{i} a_{i+1}=f(\boldsymbol{\alpha})
$$

where $f$ is given by Eq. (11).

These amendments do not affect our main results; our monogamy relations still hold true and they are tight. 